\title{
TERMOBAROMETRIA DE METAPELITOS GRANADÍFEROS DO GRUPO RIO DOCE, ORÓGENO ARAÇUAÍ
}

\author{
Tiago Amâncio Novo ${ }^{1}$, Antônio Carlos Pedrosa Soares ${ }^{1}$, Reik Degler ${ }^{1}$, Bernhard Schulz ${ }^{2}$ \\ 1 - CPMTC-IGC-UFMG, Campus Pampulha, 31270-901 Belo Horizonte, MG; tiagoanovo@gmail.com \\ 2 - Institute für Werkstoffwissenschaft, Freiberg, Alemanha
}

Recebido em 18 de maio de 2015; aceito em 30 de julho de 2015

\begin{abstract}
Resumo: O Grupo Rio Doce consiste numa unidade metavulcanossedimentar dominada por metapelitos e metawackes, com intercalações de rochas calciossilicáticas, metavulcânicas e metavulcanoclásticas que gradam para pacotes quartzíticos no topo. Caracteriza bacia relacionada ao Arco Magmático Rio Doce - Orógeno Araçuaí - com gênese e metamorfismo associados à Orogênese Brasiliano/Pan Africano. O conjunto de metapelitos da base do Grupo Rio Doce possui paragênese granadífera cristalizada durante fase deformacional principal (ca.570Ma). Os porfiroblastos de granada mostram perfis de zoneamento de Fe-Ca-Mg-Mn bem desenvolvidos caracterizados por aumento de almandina e piropo e redução dos termos grossulária e espersartita do centro para borda. Os valores P-T calculados para núcleo e borda das granadas descreve um trend de sentido horário, envolvendo aumento de temperatura (de ca. $450^{\circ} \mathrm{C}$ para $580^{\circ} \mathrm{C}$ ) sob descompressão (de ca. $8.0 \mathrm{kbar}$ para $5.8 \mathrm{kbar}$ ). Tal desenvolvimento pode estar associado à fase deformacional principal do Orógeno Araçuaí com descompressão e aumento da temperatura associados à quebra da placa subductada.
\end{abstract}

Palavras-chave: Orógeno Araçuaí, Grupo Rio Doce, metapelitos granadíferos, geotermobarometria.

Abstract: THERMOBAROMETRY OF GARNET METAPELITES FROM RIO DOCE GROUP, ARAÇUAÍ OROGEN. The Rio Doce Group consists of a metavolcanosedimentary unit composed by metapelites and metawackes interlayered with calc-silicate rocks, metavolcanic and metavolcaniclastic harrowing to quartzite packages at the top. It's an arc-related basin associated to the Rio Doce magmatic arc - Araçuaí orogeny- that underwent metamorphic and magmatic evolution during the Neoproterozoic-Early Paleozoic time. The garnet-bearing assemblages crystallized in course of the deformation stage which led to the formation of the main foliation at ca.570 Ma. The garnet porphyroblasts display well developed growth zonation of Fe-Mg-Ca-Mn and are characterized by an increase of almandine and pyrope while grossular and spessartine decrease from core to rim. The P-T values calculated from cores and rims of garnets align along clockwise trends, involving an increasing temperature (from ca. $450^{\circ} \mathrm{C}$ up to $580^{\circ} \mathrm{C}$ ) under decompression conditions (from ca. $8.0 \mathrm{kbar}$ to $5.8 \mathrm{kbar}$ ). Such development may be linked to the Araçuaí orogen main deformation phase with decompression and temperature increased associated with a slab-breakoff.

Keywords: Araçuaí orogen, Rio Doce Group, garnet metapelites, geothermobarometry.

\section{INTRODUÇÃO}

O Grupo Rio Doce é uma unidade metavulcanossedimentar associada ao arco magmático homônimo, situado no domínio interno do Orógeno Araçuaí (Alkmim et al. 2006, 2007; Brito Neves et al. 1999; Campos Neto 1995; Heilbron et al. 2004, 2008; Heilbron \& Machado 2003; PedrosaSoares et al. 1998, 2001, 2007, 2011; Trouw et al. 2013; Vieira 2007; Novo 2013). Tem deposição, deformação e metamorfismo associados a diferentes etapas do evento Brasiliano. Regionalmente, o Grupo Rio Doce inclui duas grandes sucessões: na base, uma associação dominada por metapelitos e metawackes, com intercalações de rochas calciossilicáticas, metavulcânicas e metavulcanoclásticas (formações Palmital do Sul, Tumiritinga e São Tomé), e, no topo, sustentando partes altas do relevo, pacotes ricos em quartzito (Formação João Pinto). Uma detalhada abordagem da evolução do conhecimento sobre o Grupo Rio Doce encontra-se em Vieira (2007), cujo ordenamento litoestratigráfico é aqui adotado.

Este artigo objetiva o registro de trajetórias geotermobarométricas, com identificação de episódios de crescimento da granada durante o

doi:10.18285/geonomos.v23i1.658 metamorfismo que afetou os metapelitos da sucessão basal do Grupo Rio Doce. Informações como essas, além de permitirem um avanço no entendimento da evolução termobarométrica do Orógeno Araçuaí, fornecem subsídios para testar diferentes interpretações tectônicas propostas.

\section{CONTEXTO GEOLÓGICO}

O Grupo Rio Doce configura bacia relacionada ao arco magmático do Orógeno Araçuaí, desenvolvida durante o Neoproterozoico na porção central do Gondwana ocidental, entre os crátons do São Francisco e Congo (Figura 1). A contraparte africana, no Congo, Angola, Namíbia e Gabão é representada pelo cinturão orogênico Oeste Congo. A convergência resultou em vários episódios diacrônicos do evento Brasiliano/Pan-africano, com desenvolvimento principal durante 0 Neoproterozoico-Cambriano, com últimos estágios tectônicos chegando ao Ordoviciano. O Orógeno Araçuaí-Oeste Congo apresenta uma série de características peculiares, caracterizando cinturão confinado entre os crátons São Francisco e Congo, com faixa ofiolítica neoproterozoica e grande volume de rochas plutônicas e vulcânicas relacionadas ao arco (Bilal et al. 2000; De Campos et al. 2004; Gonçalves et al. 2014; Nalini-Junior et al. 2000, 2005, 2008; Noce et al. 2000, 2006; Novo et al. 
2010; Pedrosa-Soares \& Wiedemann-Leonardos 2000, Pedrosa-Soares et al. 2001, 2008; Petitgirard et al. 2009, Queiroga et al. 2012, Queiroga 2010; Silva et al. 2002, 2005, 2008; Sollner et al. 1989; Tedeschi 2013; Vieira 2007). Sua evolução é, portanto, associada a espalhamento de fundo oceânico com subducção em natureza confinada (Alkmim et al. 2006, 2007; Pedrosa-Soares et al. 1998, 2001, 2007).

Na região abordada (Figura 1), o Grupo Rio Doce está representado pelas formações Palmital do Sul, basal, e São Tomé e João Pinto, no topo. A Formação Palmital do Sul (juntamente com a Formação Tumiritinga que ocorre a norte da área) representa a cobertura vulcano-sedimentar do Arco Rio Doce. Esta formação grada vertical e lateralmente para a Formação São Tomé que é um pacote exclusivamente sedimentar dominado por wackes e pelitos.

A Formação Palmital do Sul é composta de xistos a gnaisses, com intercalações de quartzito micáceo e/ou arcoseano, rocha calciossilicática, e rocha metavulcânica (Carvalho \& Pereira 2000, Pereira \& Zucchetti 2000, Vieira 2007). Os xistos/gnaisses são constituídos de quartzo (20-60\%), plagioclásio (0$40 \%)$, biotita (10-60\%), muscovita (0-35\%), microclina $(0-25 \%)$, granada $(0-25 \%)$, sillimanita $(0-$ $25 \%)$ e estaurolita (0-10\%). A paragênese sincinemática à foliação regional ( $\mathrm{Sn}$ ) geralmente inclui granada, estaurolita e sillimanita fibrosa, caracterizando predomínio da fácies anfibolito baixo a médio. $O$ protolito predominante é interpretado como pelito grauvaquiano. A abundância de plagioclásio nos xistos Palmital do Sul sugere fontes ricas em rochas de composição granodioritotonalito-diorito (Vieira 2007). O vulcanismo registrado na Formação Palmital do Sul é do tipo piroclástico, dacítico a riolítico, cálcio-alcalino de médio a alto potássio (Vieira 2007, Gonçalves et al. 2010). A datação U-Pb em zircão de um metatufo dacítico da Formação Palmital do Sul resultou em idade de cristalização magmática de $584 \pm 5 \mathrm{Ma}$ (Vieira 2007).

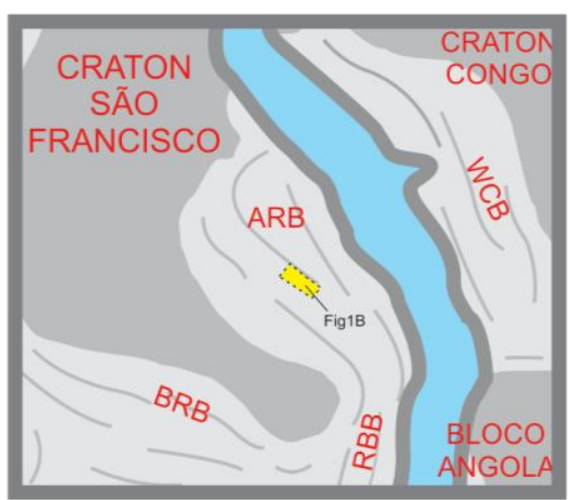

Neoproterozóico

Granitos a duas micas

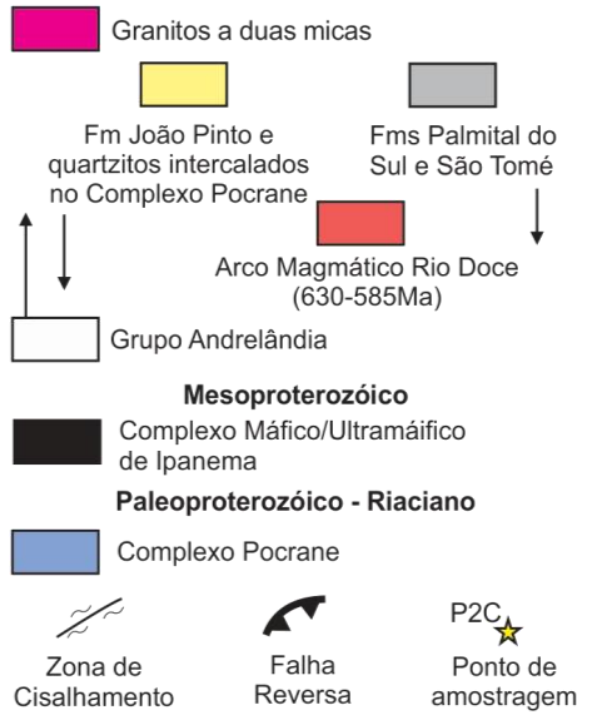



Figura 1. localização da área estudada no contexto do Gonwana Ocidental (Frimmel et al. 2011) e detalhe na região enfocada no Orógeno Araçuaí. $A R B=$ Orógeno Aracuaí; $B R B=$ Faixa Brasília; $R B B=$ Faixa Ribeira; $W C B=$ Faixa Oeste Congo. 


\section{MÉTODOS ANALÍTICOS}

As análises quantitativas de porfiroblastos de granada coexistentes com biotita e plagioclásio de dois xistos granadíferos da Formação Palmital do Sul (amostras P2C e P2E; localização apresentada na Figura 1), foram realizados com uma microssonda eletrônica JEOL JXA8900 RL no Institute für Werkstoffwissenschaft em Freiberg - Alemanha. O feixe de elétrons foi fixado em $15 \mathrm{kV} / 20 \mathrm{nA}$ e as correções ZAF matriz comum foram aplicadas. Os elementos analisados foram $\mathrm{Si}, \mathrm{Ti}, \mathrm{Al}, \mathrm{Fe}, \mathrm{Mn}, \mathrm{Mg} \mathrm{Ca}$, $\mathrm{Na}$ e K, utilizando padrões de wollastonita, rutilo, granada, hematita, bustamita, diopsídio, albita e ortoclásio. Os cristais de granada foram analisados ao longo de perfis com espaçamento de 0,05 a 0,15 $\mathrm{mm}$ por ponto. Biotita e plagioclásio foram caracterizados por análise em núcleos e borda.

Condições de temperatura e pressão foram estimadas usando: a) AVPT (média P - T; Powell \& Holland 2008), um método otimizado do Thermocalc 3,2 (Powell et al. 1998); b) termômetro granadabiotita de Bhattacharya et al. (1992) em combinação com calibração linearizada do barômetro GASP (Garnet alumino Silicate Plagioclase), com base em consistente conjunto de dados termodinâmico (Holland \& Powell 1990; Powell \& Holland 1994). Os modelos de atividade para granada dada por Ganguly et al. (1996) e para plagioclásio como proposto por Powell \& Holland (1993); e c) termometria convencional com calibrações por Thompson (1976), Holdoway \& Lee (1977), Hodges \& Spear (1982) e Perchuk \& Lavrent`eva (1983).

\section{PETROGRAFIA E GEOTERMOBAROMETRIA}

As amostras analisadas possuem porfiroblastos de granada de até $0,45 \mathrm{~cm}$ de comprimento, envoltos por matriz fina a média com cristais de quartzo e biotita alongados segundo a foliação principal (Figuras $2 \mathrm{~A}$ e $2 \mathrm{~B}$ ). A rocha possui coloração escura, reflexo do elevado teor de biotita que forma aglomerados micáceos. A mineralogia principal dos metapelitos é dada por quartzo, plagioclásio, microclima, biotita, muscovita, granada com menores proporções de estaurolita e sillimanita fibrosa (Figuras 2C e 2D). Apatita, monazita, zircão e minerais opacos são os acessórios comuns. Carbonato e sericita são os principais produtos de alteração de plagioclásio. O granada-biotita xisto exibe uma foliação (Sn) bem desenvolvida, comumente dobrada e crenulada. Os minerais índices, como granada, estaurolita e sillimanita, marcam a xistosidade regional, sendo sincinemáticas à deformação principal. Os cristais de granada encontram-se parcialmente alterados para biotita mostrando inclusões de quartzo, biotita, plagioclásio e minerais opacos. A foliação de crenulação $\left(S_{n+1}\right)$ é marcada essencialmente por biotita, podendo estar relacionada a níveis crustais mais rasos.
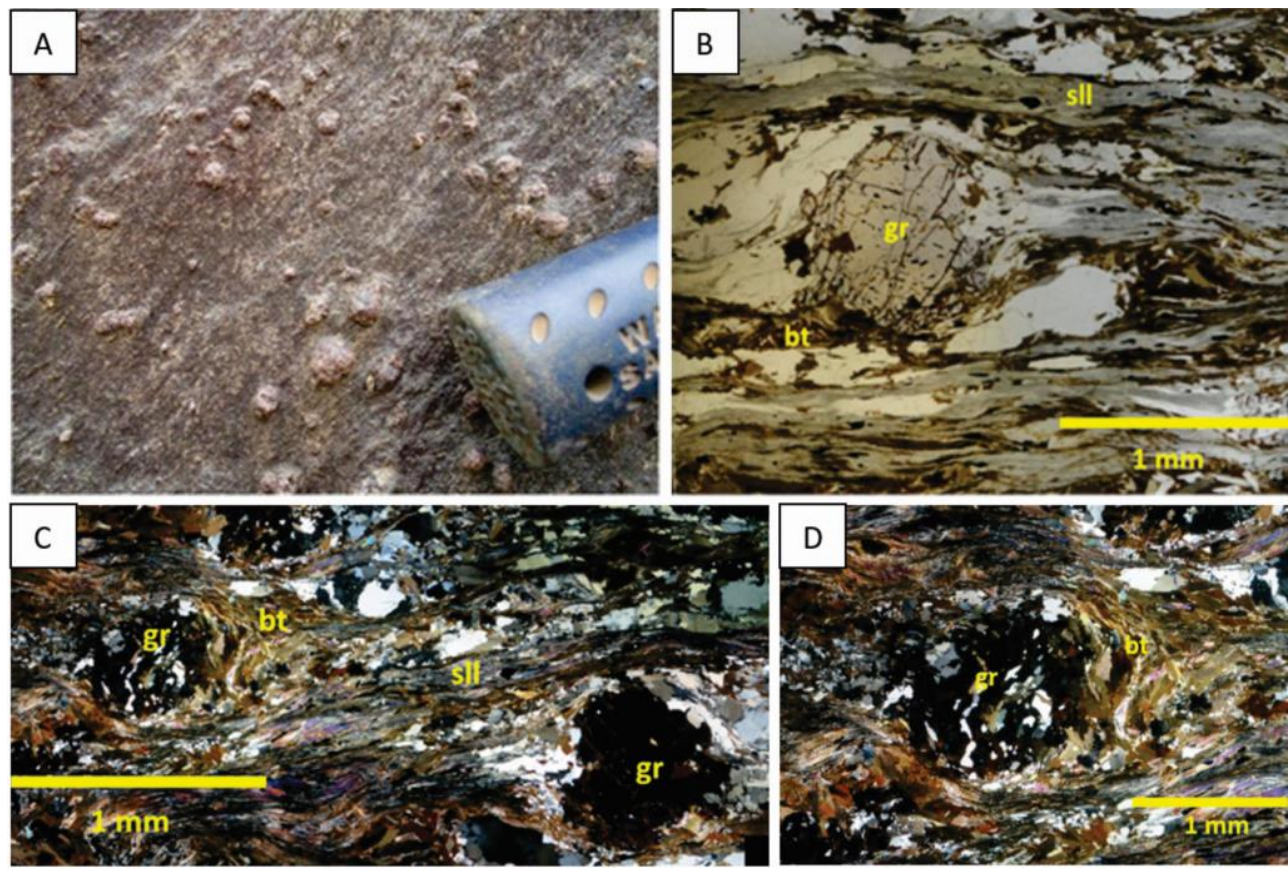

Figura 2. Feições do xisto granadífero da Formação Palmital do Sul. A) granada-biotita xisto com foliação penetrativa (amostra P2C); B) porfiroblasto de granada envolto pela foliação dada por sillimanita e biotita (nicóis paralelos - amostra P2C); C) sillimanita-granada-biotita xisto, destaque para sillimanita fibrosa estirada segundo Sn (nicóis cruzados - amostra P2E); D) poiquiloblasto de granada rotacionado, rico em inclusões de minerais félsicos e biotita (nicóis cruzados - amostra P2C). 

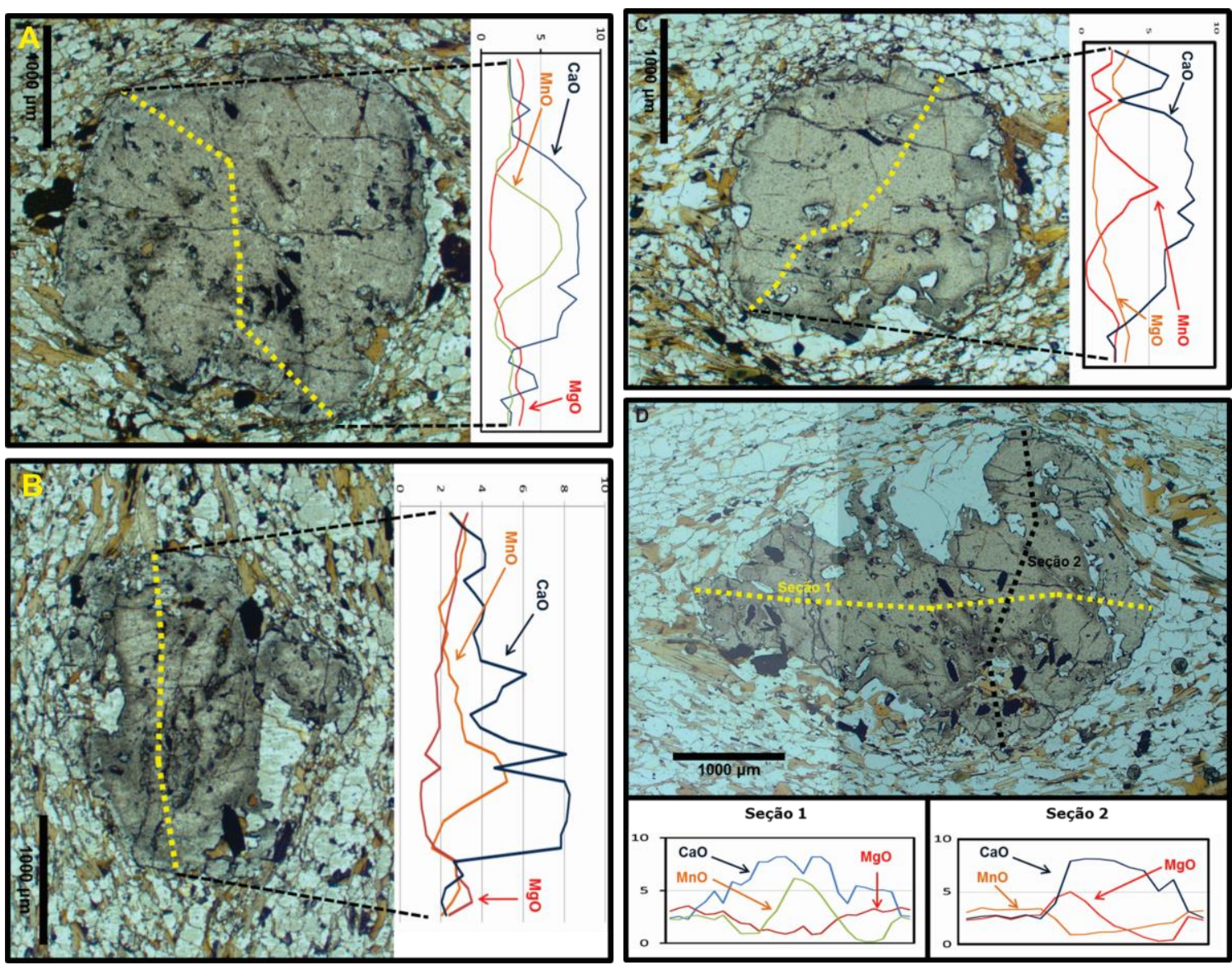

Figura 3. Porfiroblastos de Granada das amostras P2C (A e B) e P2E (C e D) mostrando perfis analisados (borda-núcleo-borda) com zoneamento em Piropo ( $\mathrm{MgO}$ ), Grossulária (CaO) e Espersatita (MnO).

Boa parte dos minerais do grupo da granada são soluções sólidas entre dois ou mais membros finais, cuja fórmula estrutural pode ser descrita por $\mathrm{X}_{3}{ }^{[8]} \mathrm{Y}_{2}{ }^{[6]}\left[\mathrm{SiO}_{4}\right]_{3}$, onde $\mathrm{X}=\mathrm{Mg}^{2+}, \mathrm{Fe}^{2+}, \mathrm{Mn}^{2+}$ ou $\mathrm{Ca}^{2+}$ e $\mathrm{Y}$ $=\mathrm{Al}^{3+}, \mathrm{Fe}^{3+}, \mathrm{Ti}^{4+}, \mathrm{Cr}^{3+}$ ou $\mathrm{V}^{3+}$. Análise de granadas dos metapelitos da Fm. Palmital do Sul mostram correlação negativa entre $\mathrm{FeO}+\mathrm{MnO}$ versus $\mathrm{CaO}+\mathrm{MgO}$ sugerindo contexto onde as principais substituições catiônicas tendem a envolver os íons ferro, magnésio, manganês e cálcio (Figura 4A). A dispersão entre os óxidos $\mathrm{FeO}$ e $\mathrm{MgO}$ mostra correlação positiva (figura 4B), evidenciando ausência de fracionamento entre os íons $\mathrm{Fe}^{2+}$ e $\mathrm{Mg}^{2+}$. Com isso o comportamento químico destas granadas é controlado basicamente por substituições do tipo $\mathrm{Mn}^{2+}+\mathrm{Ca}^{2+} \rightarrow 2 \mathrm{Mg}^{2+} \quad$ (evidenciado pela correlação negativa, Figura $4 \mathrm{C}$ ).

O perfil de zonamento nos porfiroblastos de granada é caracterizado pelo decréscimo dos termos espersatita (16,3\% para $2,4 \%$ do end member) e grossulária (19,7\% para $5,9 \%$ do end member) do centro para a borda (Figura 3; Tabela 1). Piropo tem mínimo de 1,8\% no núcleo dos cristais, aumentando para até $8,1 \%$ na borda. Comparativamente o termo almandina sempre apresenta os maiores valores, também crescentes do centro para borda $(62,1 \%$ para $81,3 \%)$. O zoneamento da granada é caracterizado por aumento de Mg e Fe e redução de Mn e Ca do centro para a borda (Figura 3; Tabela 1), implicando num padrão metamórfico progressivo. 0 aumento do termo piropo do núcleo para borda da granada tende a refletir um decréscimo na concentração de magnésio da biotita $(\mathrm{Mg} / \mathrm{Mg}+\mathrm{Fe}$; 0.3997 - 0.4220) A forte variação do termo grossulária é balanceada em direção oposta à evolução do cálcio no plagioclásio. Quanto maior a taxa de difusão de plagioclásio após inclusão em granada, maior será o empobrecimento em cálcio do plagioclásio (Figura 5; incluso na granada ocorre termo bytownita e na borda andesina). Ou seja, as variações químicas encontradas nos cristais de plagioclásio inclusos em granadas e dispersos na matriz refletem o equilíbrio químico das diferentes etapas de crescimento da granada. 
Tabela 1. Síntese das análises de granada (Grt), biotita (Bt) e plagioclásio (PI) dos metapelitos granadíferos em microssonda eletrônica, normalizados para 120 (granada), 220 (biotita) e 80

(plagioclásio). As análises minerais combinadas para cálculos geotermobarométricos são os seguintes: Amostra P2E:

Grt1CBt1PII; Grt1BBt2PI2; Grt2CBt3PI3; Grt2BBt4PI4; Grt3CBt5PI5; Grt3BBt6PI6; Amostra P2C: Grt4CBt7PI7; Grt4BBt8PI8;

Grt5CBt9P19; Grt5BBt10PI10. (Alm = almandina; Prp = piropo; Sps = espessartita; $G r s=$ grossulária; $N=$ núcleo; $B=$ borda).

\begin{tabular}{ccccccc}
\hline \multirow{2}{*}{ Grt } & \multicolumn{2}{c}{ P2E-1 } & \multicolumn{2}{c}{ P2E-2 } & \multicolumn{2}{c}{ P2E-3 } \\
\cline { 2 - 7 } & P2E-1C & P2E-1B & P2E-2C & P2E-2B & P2E-3C & P2E-3B \\
\hline Si & 3,820 & 3,846 & 3,829 & 3,854 & 3,780 & 3,802 \\
Al & 2,012 & 2,099 & 2,020 & 2,071 & 1,990 & 2,086 \\
Fe & 2,962 & 3,322 & 2,846 & 3,328 & 2,925 & 3,231 \\
Mn & 0,306 & 0,224 & 0,412 & 0,232 & 0,363 & 0,099 \\
Mg & 0,103 & 0,333 & 0,093 & 0,306 & 0,115 & 0,233 \\
Ca & 0,818 & 0,253 & 0,818 & 0,245 & 0,835 & 0,589 \\
Tot. & 10,035 & 10,084 & 10,037 & 10,043 & 10,029 & 10,045 \\
Alm & 70,7 & 80,4 & 68,2 & 80,9 & 69,0 & 77,8 \\
Prp & 2,5 & 8,1 & 2,2 & 7,4 & 2,7 & 5,6 \\
Sps & 7,3 & 5,4 & 9,9 & 5,6 & 8,6 & 2,4 \\
Grs & 19,5 & 6,1 & 19,6 & 6,0 & 19,7 & 14,2
\end{tabular}

\begin{tabular}{ccccccc}
\hline Bt & 1 & 2 & 3 & 4 & 5 & 6 \\
\hline Si & 3,644 & 3,696 & 3,636 & 3,731 & 3,715 & 3,682 \\
Ti & 0,187 & 0,167 & 0,171 & 0,166 & 0,174 & 0,175 \\
Al & 1,901 & 1,902 & 1,895 & 1,900 & 1,901 & 1,917 \\
Fe & 1,637 & 1,631 & 1,681 & 1,644 & 1,704 & 1,688 \\
Mn & 0,002 & 0,002 & 0,001 & 0,000 & 0,000 & 0,001 \\
Mg & 1,160 & 1,152 & 1,137 & 1,136 & 1,115 & 1,127 \\
Ca & 0,001 & 0,000 & 0,001 & 0,000 & 0,001 & 0,001 \\
Na & 0,026 & 0,025 & 0,028 & 0,025 & 0,025 & 0,022 \\
K & 0,915 & 0,923 & 0,905 & 0,915 & 0,919 & 0,875 \\
Tot. & 9,478 & 9,501 & 9,459 & 9,520 & 9,558 & 9,491
\end{tabular}

\begin{tabular}{ccccccc}
\hline PI & 1 & 2 & 3 & 4 & 5 & 6 \\
\hline Si & 6,020 & 5,784 & 6,109 & 6,312 & 6,046 & 5,133 \\
Ti & 0,000 & 0,004 & 0,002 & 0,000 & 0,000 & 0,000 \\
Al & 2,429 & 2,525 & 2,374 & 2,230 & 2,448 & 2,910 \\
Fe & 0,018 & 0,039 & 0,005 & 0,002 & 0,026 & 0,031 \\
Mn & 0,001 & 0,005 & 0,000 & 0,000 & 0,000 & 0,003 \\
Mg & 0,000 & 0,000 & 0,000 & 0,001 & 0,000 & 0,000 \\
Ca & 0,695 & 0,826 & 0,625 & 0,456 & 0,672 & 1,314 \\
Na & 0,825 & 0,753 & 0,867 & 0,982 & 0,814 & 0,438 \\
K & 0,005 & 0,008 & 0,007 & 0,008 & 0,003 & 0,004 \\
Tot. & 9,996 & 9,947 & 9,991 & 9,993 & 10,012 & 9,836
\end{tabular}

\begin{tabular}{ccccc}
\hline Grt & \multicolumn{2}{c}{$P 2 C-1$} & \multicolumn{2}{c}{$P 2 C-2$} \\
\cline { 2 - 5 } & P2C-4C & P2C-4B & P2C-5C & P2C-5B \\
\hline Si & 3,826 & 3,824 & 3,837 & 3,823 \\
Al & 2,007 & 2,077 & 2,006 & 2,068 \\
Fe & 2,573 & 3,329 & 2,785 & 3,260 \\
Mn & 0,675 & 0,218 & 0,522 & 0,316 \\
Mg & 0,075 & 0,303 & 0,105 & 0,303 \\
Ca & 0,818 & 0,242 & 0,802 & 0,301 \\
Tot. & 9,993 & 10,00 & 10,07 & 10,07 \\
Alm & 62,1 & 81,3 & 66,0 & 77,9 \\
Prp & 1,8 & 7,4 & 2,5 & 7,2 \\
Sps & 16,3 & 5,3 & 12,4 & 7,6 \\
Grs & 19,7 & 5,9 & 19,0 & 7,2
\end{tabular}

\begin{tabular}{ccccc}
\hline $\mathbf{B t}$ & $\mathbf{7}$ & $\mathbf{8}$ & $\mathbf{9}$ & $\mathbf{1 0}$ \\
\hline $\mathbf{S i}$ & 3,658 & 3,405 & 3,732 & 3,730 \\
$\mathbf{T i}$ & 0,182 & 0,157 & 0,185 & 0,146 \\
$\mathbf{A l}$ & 1,924 & 1,860 & 1,903 & 1,919 \\
$\mathbf{F e}$ & 1,639 & 1,587 & 1,674 & 1,632 \\
$\mathbf{M n}$ & 0,000 & 0,001 & 0,004 & 0,004 \\
$\mathbf{M g}$ & 1,091 & 1,158 & 1,128 & 1,145 \\
$\mathbf{C a}$ & 0,002 & 0,003 & 0,001 & 0,002 \\
$\mathbf{N a}$ & 0,023 & 0,023 & 0,027 & 0,023 \\
$\mathbf{K}$ & 0,869 & 0,706 & 0,911 & 0,912 \\
Tot. & 9,391 & 8,904 & 9,569 & 9,518
\end{tabular}

\begin{tabular}{ccccc}
\hline $\mathbf{P I}$ & 7 & 8 & 9 & 10 \\
\hline $\mathbf{S i}$ & 6,339 & 6,132 & 6,234 & 6,112 \\
$\mathrm{Ti}$ & 0,002 & 0,001 & 0,000 & 0,000 \\
$\mathbf{A l}$ & 2,233 & 2,396 & 2,334 & 2,392 \\
$\mathbf{F e}$ & 0,011 & 0,029 & 0,000 & 0,016 \\
$\mathbf{M n}$ & 0,000 & 0,000 & 0,001 & 0,001 \\
$\mathbf{M g}$ & 0,000 & 0,000 & 0,000 & 0,000 \\
$\mathbf{C a}$ & 0,450 & 0,624 & 0,563 & 0,625 \\
$\mathbf{N a}$ & 0,971 & 0,858 & 0,903 & 0,883 \\
$\mathbf{K}$ & 0,006 & 0,005 & 0,005 & 0,003 \\
Tot. & 10,016 & 10,049 & 10,043 & 10,035
\end{tabular}
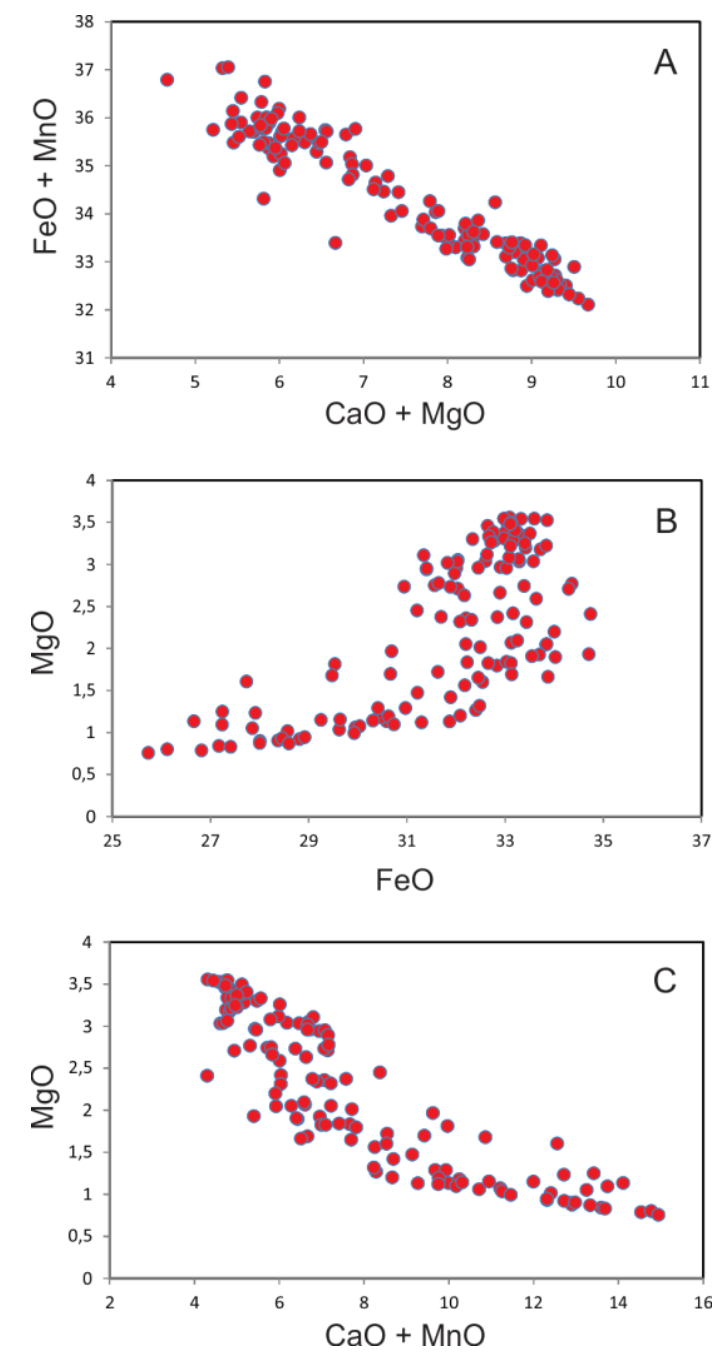

Figura 4. Diagramas binários para teores de $\mathrm{FeO}, \mathrm{MnO}, \mathrm{CaO} e$ $\mathrm{MgO}$ de granadas dos metapelitos da Fm. Palmital do Sul. A) correlação negativa da distribuição de (CaO+MnO) e (FeO+MgO); B) correlação positiva na distribuição de FeOt e $\mathrm{MgO}$ e; C) correlação negativa entre $(\mathrm{CaO}+\mathrm{MnO})$ e $\mathrm{MgO}$. 


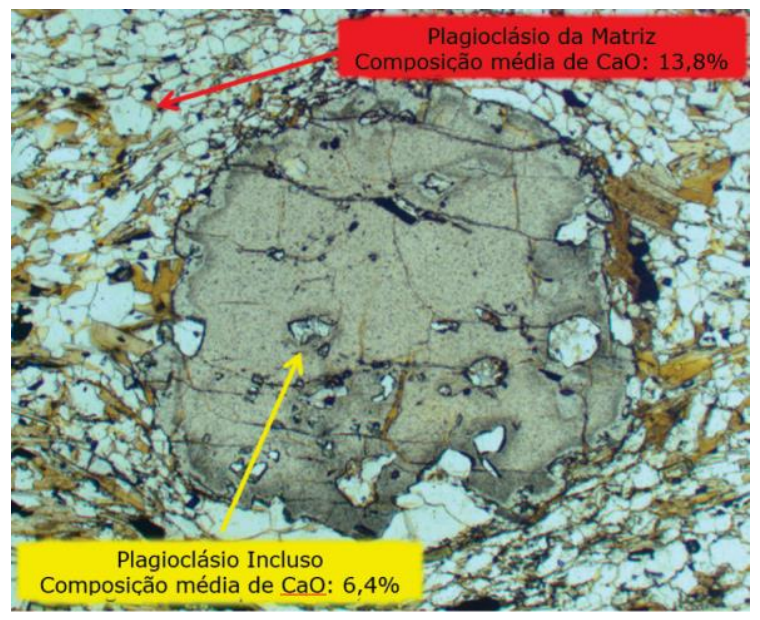

Figura 5. Cristal de granada da amostra $P 2 E$, com destaque para a composição média de $\mathrm{CaO}$ em cristais de plagioclásio incluso na granada e disperso na matriz

Geotermobarometria baseada na assembleia mineralógica de granadas zonadas revelou evolução PT num sentido horário com aumento da temperatura $\left(442^{\circ} \mathrm{C}\right.$ a $\left.590^{\circ} \mathrm{C}\right)$ e descompressão (9,3Kbar a 4,2Kbar) do núcleo para borda (Figura 6; Tabela 2), correspondendo à fácies anfibolito baixa a média. Os valores PT obtidos estão de acordo com as condições metamórficas descritas para o Grupo Rio Doce na região (Vieira 2007; Novo 2013). Os cálculos geotermobarométricos possuem um erro mínimo de $\pm 50^{\circ} \mathrm{C}$ e $\pm 1 \mathrm{kbar}$ relacionados a erros da microssonda e imprecisões de dados termodinâmicos envolvidos nas calibrações.

Tabela 2. dados geotermobarométricos para os metapelitos granadíferos da Fm. Palmital do Sul. Termobarometria convencional com Biotita + Granada (Bhattacharya et al., 1992) e GASP (Powell and Holland, 1993).

\begin{tabular}{ccccc} 
& & Núcleo & Borda & \\
\cline { 2 - 5 } Amostra & T oC & P Kbar & T oC & P Kbar \\
\hline $\begin{array}{c}\text { P2C } \\
\text { (Granada 1) }\end{array}$ & 448 & 8,2 & 568 & 5,4 \\
$\begin{array}{c}\text { P2C } \\
\text { (Granada 2) }\end{array}$ & 442 & 9,4 & 588 & 6,6 \\
\hline $\begin{array}{c}\text { P2E } \\
\text { (Granada 1) }\end{array}$ & 450 & 6,5 & 577 & 6,8 \\
$\begin{array}{c}\text { P2E } \\
\text { (Granada 2) }\end{array}$ & 465 & 5,9 & 583 & 5,8 \\
$\quad$ P2E & 477 & 6,6 & 590 & 4,2 \\
(Granada 3) & & & &
\end{tabular}

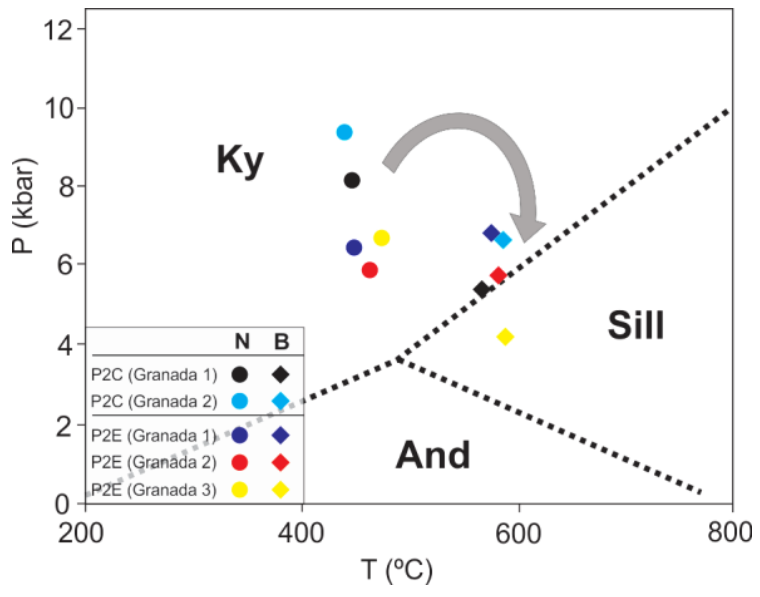

Figura 6. dados PT e trend PT para os metapelitos granadiferos. Resultados calculados com termobarometria convencional com Biotita + Granada (Bhattacharya et al., 1992) e GASP (Powell and Holland, 1993). Campos de estabilidade de Cianita (Ky), Andalusita (And) e Sillimanita (Sil) dados pela orientação das coordenadas PT (Spear 1993). N = núcleo; $B=$ borda.

\section{CONCLUSÃO}

Condições de temperatura e pressão foram determinadas com base em numerosos perfis em granada e interpretados de modo a refletir a evolução metamórfica local da Fm. Palmital do Sul. Todas as amostras possuem foliação penetrativa, com porfiroblastos de granada envoltos em matriz biotítica de granulação fina a média. De acordo com as microestruturas, os grandes cristais de granada têm cristalização sin-deformacional durante o desenvolvimento da foliação $\mathrm{Sn}$ regional. Todas as granadas analisadas exibem zonamento PT com decréscimo do teor de espersartita e aumento do conteúdo de piropo e almandina do centro para a borda. Geotermobarometria foi aplicada aos núcleos e bordas de granadas com biotita e plagioclásio em equilíbrio. Os resultados termobarométricos refletem o zonamento e representam evolução no sentido horário com o aumento de temperatura e descompressão - de ca. $442{ }^{\circ} \mathrm{C}$ para 590ㄷ e ca. $9.3 \mathrm{kbar}$ para $4.2 \mathrm{kbar}$. Este tipo de padrão é muito comum em sistemas orogênicos, onde a descompressão pode ser devida a erosão ou desnudação tectônica (Winter 2001). Levando em consideração o modelo evolutivo do Orógeno Araçuaí, o aumento termal associado à descompressão pode estar relacionado à quebra de slab com soerguimento astenosférico, o que implica em underplating mantélico que usualmente sustenta elevado fluxo de calor a longo prazo. Uma segunda 
interpretação estaria associada ao colapso orogênico, quando a gênese de granitos póstectônicos garantiria o aumento da temperatura no estágio termal final.

\section{AGRADECIMENTOS}

Os autores agradecem ao CNPq, FAPEMIG, CAPES, CODEMIG e PrPq-UFMG pelos recursos financeiros à pesquisa científica, bolsas e projetos de mapeamento geológico.

\section{REFERÊNCIAS BIBLIOGRÁFICAS}

Alkmim F.F., Pedrosa-Soares A.C., Noce C.M., Cruz S.C.P. 2007 Sobre a Evolução Tectônica do Orógeno Araçuaí-Congo Ocidental. Geonomos, 15:25-43.

Alkmim F.F., Marshak S., Pedrosa-Soares A.C., Peres G.G., Cruz S.C.P., Whittington A. 2006. Kinematic evolution of the Araçuaí-West Congo orogen in Brazil and Africa: Nutcracker tectonics during the Neoproterozoic assembly of Gondwana. Precambrian Research, 149:63.82.

Battacharya A., Mohanty L., Maji, A., Sen S.K., Raith M. 1992. Non ideal mixing in the phogopiteannite boundary: constraints from experimental data on MgFe partitioning and reformulation of the biotite garnet geothermometer. Contributions to Mineralogy and Petrology, 111(1):87-93.

Bilal E., Horn H. A., Naline Jr H. A., Mello F. M., Correia-Neves J.M., Giret A. R., Moutte J., Fuizikawa K., Fernandes M. L. S. 2000. Neoproterozoic granitoid suites in southeastern Brazil. Brazilian contributions to the 31st International Geological Congress, Brazil/2000. Rev. Bras. Geoc., 30(1):51-54.

Brito-Neves B.B., Campos-Neto M.C., Fuck R.A. 1999. From Rodinia to Western Gondwana: An approach to the Brasiliano- Pan African cycle and orogenic collage. Episodes, 22:155-199.

Campos-Neto M.C., Figueiredo M.C.H., 1995. The Rio Doce Orogeny, south-eastern Brazil. Journal of South American Earth Sciences, 8:143-162.

Carvalho J.B., Pereira L.M.M. 2000. Projeto Leste: Petrografia, relatório integrado. Etapa II. Belo Horizonte: SEME/CPRM/CODEMIG, CD-ROM.

De Campos C.M., Mendes J.C., Ludka I.P., Medeiros S.R., Moura J.C., Wallfass, C. 2004. A review of the Brasiliano magmatism in southern Espírito Santo, Brazil, with emphasis on postcollisional magmatism. Journal of the Virtual Explorer, 17: 1-35.

Frimmel H.E, Basei M.S., Gaucher C. 2011. Neoproterozoic geodynamic evolution of SW-Gondwana: a southern African perspective. Int J Earth Sci (Geol Rundsch), 100: 323-354.

Ganguly J., Cheng W. \& Tirone M. 1996. Thermodynamics of aluminosilicate garnet solid solution: new experimental data, an optimized model, and thermometric applications. Contributions to Mineralogy and Petrology. 126(12):137-151.

Gonçalves L., Farina F., Lana C., Pedrosa-Soares A. C., Alkmim F., Nalini H. A. 2014. New U-Pb Ages and Lithochemical Attributes of the Ediacaran Rio Doce Magmatic Arc, Araçuaí Confined Orogen, Southeastern Brazil. Journal of South American Earth Sciences, 52:1-20.

Gonçalves L.S., Alkmim F.F., Pedrosa-Soares A.C. 2010 Características geoquímicas da Suíte G1, arco magmático do
Orógeno Araçuaí, entre Governador Valadares e Ipanema, MG. REM. Revista Escola de Minas, 63:457-464.

Heilbron M., Valeriano C. M., Tassinari C. C. G., Almeida J., Tupinamba M., Siga O., Trouw R. 2008. Correlation of Neoproterozoic terranes between the Ribeira Belt, SE Brazil and its African counterpart: comparative tectonic evolution and open questions. Geological Society Special Publication, 294:211-237.

Heilbron M.L., Pedrosa-Soares A.C., Campos Neto M.C., Silva L.C., Trouw R. \& Janasi V.A. 2004. Província Mantiqueira. In: V.M. Mantesso-Neto, A. Bartorelli, C.D.R. Carneiro \& Brito-Neves, B.B. (orgs.). Geologia do Continente Sul-Americano.São Paulo, Editora Beca, p: 203-234.

Heilbron M. \& Machado N. 2003. Timing of terrane accretion in the Neoproterozoic-Eopaleozoic Ribeira orogen (SE Brazil). Precambrian Res., 125: 87-112.

Hodges K.V. \& Spear F.S. 1982. Geothermometry, geobarometry and the Al2SiO5 triple point at Mt. Moosilauke, New Hampshire. American Mineralogist, 67:1118-1134.

Holdoway M.J. \& Lee S.M. 1977. Fe-Mg cordierite stability in highgrade pelitic rocks based on experimental, theoretical and natural observations. Contributions to Mineralogy and Petrology. 63(2):175-198.

Holland T.J.B. \& Powell R. 1990. An enlarged and updated internally consistente thermodynamic dataset with uncertainties and correlations: the system $\mathrm{K}_{2} \mathrm{O}-\mathrm{Na}_{2} \mathrm{O}-\mathrm{CaO}-$ $\mathrm{MgO}-\mathrm{MnO}-\mathrm{FeO}-\mathrm{Fe}_{2} \mathrm{O}_{3}-\mathrm{Al}_{2} \mathrm{O}_{3}-\mathrm{TiO}_{2}-\mathrm{SiO}_{2}-\mathrm{CH}_{2} \mathrm{O}_{2}$. Journal of Metamorphic Geology, 8:89-124.

Nalini Jr H. A., Machado R., Endo, I., Bilal E. 2008. A Importância da tectônica transcorrente no alojamento de granitos pré a sincolisionais na região do vale do médio Rio Doce: o exemplo das suítes Galiléia e Urucum. Revista Brasileira de Geociências, 38:748-759.

Nalini Jr H. A., Machado R., Bilal E. 2005. Geoquímica e petrogênese da Suíte Galiléia: exemplo de magmatismo tipo-I metaluminoso pré-colisional Neoproterozóico da região do médio rio Doce (MG). Revista Brasileira de Geociências. 35:23-34.

Nalini H.A., Bilal E., Paquette J.L., Pin C., Machado R. 2000. Geochronologie $\mathrm{U}-\mathrm{Pb}$ et géochimie isotopique $\mathrm{Sr}-\mathrm{Nd}$ dês granitoides neoproterozoiques des suites Galileia et Urucum, vallée du Rio Doce, Sud-Est du Brésil. Compte Rendu Academie Science Paris, 331: 459-466.

Noce, C.M., Novo T., Figueiredo, C., Pedrosa-Soares, A. C. 2006. Mapa geológico da Folha Manhuaçu (SF.23-X-B-III). Rio de Janeiro, CPRM/UFMG - Programa Geologia do Brasil, escala $1: 100.000$.

Noce C. M., Macambira M. J. B., Pedrosa-Soares A. C. 2000. Chronology of Neoproterozoic-Cambrian granitic magmatism in the Araçuaí Belt, Eastern Brazil, based on single zircon evaporation dating.. Revista Brasileira de Geociências, 30(1):25-29.

Novo T., 2013. Caracterização do Complexo Pocrane, magmatismo básico mesoproterozóico e unidades neoproterozóicas do sistema Araçuaí-Ribeira, com ênfase em geocronologia U-Pb (SHRIMP e LA-ICP-MS). Universidade Federal de Minas Gerais, Belo Horizonte, Brazil, p. 193.

Novo T., Pedrosa-Soares A.C., Noce C.M., Alkmim F.F., Dussin I. 2010. Rochas charnockíticas do sudeste de Minas Gerais: a raiz granulítica do arco Magmático do Orógeno Araçuaí. Revista Brasileira de Geociências, 40(4): 573-592.

Pedrosa-Soares A. C., Campos C., Noce C.M., Silva L.C., Roncato J., Novo T., Medeiros S., Castañeda C., Queiroga G., Dantas E., Dussin I, Alkmim F.F. 2011. Late Neoproterozoic-Cambrian 
granitic magmatism in the Araçuaí Orogen, the Eastern Brazilian Pegmatite Province and related mineral resources (SE Brazil). Geological Society London, 350:25-51.

Pedrosa-Soares A.C., Alkmim F.F., Tack L., Noce C.M., Babinski M., Silva L.C., Martins-Neto M.A. 2008. Similarities and differences between the Brazilian and African counterparts of the Neoproterozoic Araçuaí-West-Congo orogen. Geological Society, London, Special Publications, 294: 153-172

Pedrosa-Soares A. C., Noce C. M., Alkmim F. F., Silva L. C., Babinski M., Cordani U., Castañeda C. 2007. Orógeno Araçuaí: síntese do conhecimento 30 anos após Almeida 1977. Geonomos, 15: 1-16.

Pedrosa-Soares A.C., Noce C.M., Wiedemann C.M., Pinto C.P. 2001. The Araçuaí-West Congo orogen in Brazil: An overview of a confined orogen formed during Gondwanland assembly. Precambrian Research, 110: 307-323.

Pedrosa-Soares A.C. \& Wiedemann-Leonardos C.M. 2000 Evolution of the Araçuaí Belt and its connection to the Ribeira Belt, Eastern Brazil. In: U. Cordani, E. Milani, A. Thomaz-Filho \& D. A. Campos (eds), Tectonic Evolution of South America. São Paulo, Sociedade Brasileira de Geologia, p. 265-285.

Pedrosa-Soares A.C., Vidal P., Leonardos O.H., Brito-Neves B.B., 1998. Neoproterozoic oceanic remnants in eastern Brazil: Further evidence and refutation of an exclusively ensialic evolution for the Araçuaí-West Congo orogeny. Geology, 26, 519-522.

Pereira L.M.M. \& Zucchetti M. 2000. Projeto Leste: Petrografia, relatório integrado. Etapa II. Belo Horizonte: SEME/CPRM/CODEMIG, CD-ROM.

Perchuk, L.L \& Lavrent'eva, I.V. 1983. Experimental investigation of exchange equilibria in the system cordieritegarnetbiotite. In: SAXENA, S.K. ed. Kinetics and Equilibrium in Mineral Reactions, Advances in Physical Geochemistry. New York: SpringerVerlag, p.199-239.

Petitgirard S. \& Vauchez A. et al. 2009. Conflicting structural and geochronological data from the Ibituruna quartz-syenite (SE Brazil): Effect of protracted 'hot' orogeny and slow cooling rate? Tectonophysics, 477: 174-196.

Powell R. \& Holland T.J.B. 2008. On thermobarometry. Journal of Metamorphic Geology, 26:155-179.

Powell R., Holland T.J.B., Worley B. 1998. Calculating phase diagrams involving solid solutions via nonlinear equations, with examples using THERMOCALC. Journal of Metamorphic Geology, 16:577-588.

Powell R. \& Holland T.J.B. 1994. Optimal geothermometry and geobarometry. American Mineralogist, 79:120-133.

Powell R. \& Holland T.J.B. 1993. On the formulation of simple mixing models for complex phases. American Mineralogist, 78:1174-1180
Queiroga G., Suita M.T.F., Pedrosa-Soares A.C., Martins M., Pinheiro M.A.P. 2012. Síntese sobre ofiolitos: evolução dos conceitos. REM. Revista Escola de Minas (Impresso), 65:4758.

Queiroga G.N. 2010. Caracterização de restos de litosfera oceânica do Orógeno Araçuaí entre os paralelos 17으 e 21으 S. Tese de Doutorado, Instituto de Geociências, Universidade Federal de Minas Gerais, 180 p.

Silva L.C., Pedrosa-Soares A.C., Teixeira L., Armstrong R. 2008. Tonian rift-related, A-type continental plutonism in the Araçuaí Orogen, eastern Brazil: New evidence for the breakup stage of the São Francisco Congo Paleocontinent. Gondwana Research, 13:527-537.

Silva L.C., McNaughton, N.J., Armstrong, R., Hartmann, L. \& Fletcher, I. 2005. The Neoproterozoic Mantiqueira Province and its African connections. Precambrian Research, 136: 203240.

Silva L.C., Armstrong R, Noce C.M., Carneiro M., Pimentel M., Pedrosa-Soares A. C., Leite C., Vieira V. S., Silva M., Paes V., Cardoso-Filho J. 2002. Reavaliação da evolução geológica em terrenos pré-cambrianos brasileiros com base em novos dados U-Pb SHRIMP, parte II: Orógeno Araçuaí, Cinturão Móvel Mineiro e Cráton São Francisco Meridional. Revista Brasileira de Geociências, 32: 513-528.

Söllner F., Lammerer B., Weber-Diefenbach K., 1989. Brasiliano age of a charnoenderbitic rock suite in the Complexo Costeiro (Ribeira Mobile Belt), Espirito Santo, Brazil: evidence from U$\mathrm{Pb}$ geochronology on zircons. Zentralblatt $\mathrm{f}$. Geologie und Paleontologie, Teil I, 5(6):933-943

Spear F. 1993 Metamorphic phase equilibria and pressure temperature time paths. Mineralogical Society of America, Monograph, Series 1, 799p.

Tedeschi M. 2013. Caracterização do Arco Magmático do Orógeno Araçuaí entre Frei Inocêncio e Itambacuri, MG. Dissertação de Mestrado, Instituto de Geociências, Universidade Federal de Minas Gerais, 89p.

Thompson, A. B. 1976. Mineral reactions in pelitic rocks: II. Calculation of some P-T-X (Fe-Mg) phase relations. American Journal of Science, 276:401-454.

Trouw, R.A.J., Peternel, R., Ribeiro, A., Heilbron, M., Vinagre, R., Duffles, P., Trouw, C. C., Fontainha, M., Kussama, H. H., 2013. A new interpretation for the interference zone between the southern Brasília belt and the central Ribeira belt, SE Brazil. Journal of South American Earth Sciences, 48:43-57.

Vieira V.S. 2007. Significado do Grupo Rio Doce no Contexto do Orógeno Araçuaí. Tese de Doutorado, Instituto de Geociências, Universidade Federal de Minas Gerais, 117p.

Winter O.D. 2001. An introduction to Igneous and Metamorphic Petrology. New Jersey: Prentice Hall, 697p. 